\title{
COMMISSION 21: LIGHT OF THE NIGHT SKY
} (LUMIERE DU CIEL NOCTURNE)

\author{
PRESIDENT: Philippe Lamy \\ VICE-PRESIDENT: J. Mayo Greenberg ${ }^{\dagger}$ \\ ORGANIZING COMMITTEE: S. Bowyer, I. Mann, B.Å.S. Gustafson, \\ A-C. Levasseur-Regourd, T. Mukai, S. Price, W. Reach \& M. Hanner
}

$\dagger$ Deceased Nov 29, 2001

Ground based observations of the Zodiacal Light in the visible are actively pursued by the group at Kobe University (Japan), led by T. Mukai. Using large-angle optics combined with CCD detector, their imaging program aims at characterizing the structure of the Zodiacal Light and its various components: dust trails, libration clouds, etc. See articles by Mukai et al., Urakawa et al., Ishiguro et al., and Takahashi et al. - all published in the Proceedings of IAU Coll. 181 (also COSPAR Coll. Series 15), "Dust in the Solar System and Other Planetary Systems" (Eds. S.F. Green et al., Pergamon) - and Ishiguro et al. (Ap.J. 572, L117-120, 2002).

As part of a program to study the extragalactic background light (EBL) at 3000, 5000 and $8000 \AA$, the seasonal variations of the Zodiacal Light toward a fixed extragalactic target at high ecliptic latitude have been monitored. The required accuracy to reach the EBL and systematic errors have been discussed (Bernstein et al., Ap.J. 571, 85-106, 2002).

Space observations of the Zodiacal Light were performed in the mid IR by ISOPHOT. Spectra are featureless and temperature variations can be explained by the 3-D geometry of a well-mixed interplanetary dust cloud (Leinert et al., A\&A 393, 1073-1079, 2002).

The inner Zodiacal Light has been studied from the Clementine spacecraft using the Moon to occult the Sun. The 2-D brightness map is interpreted in terms of three dust populations having different inclinations (Hahn et al., Icarus 158, 360-378, 2002).

Numerical models of the dust bands that incorporate large (100 microns) particles evidenced in Long Duration Exposure Facility (LDEF) cratering records have been developed and are able to match both the shapes and amplitudes observed by IRAS in multiple wavebands (Grogan et al., Icarus 152, 251-267, 2001).

Radiation pressure and the Poynting-Robertson effect for fluffy dust particles have been investigated. The $\mathrm{P}-\mathrm{R}$ effect alone was found insufficient for giving a satisfactory estimate of their lifetimes (Kimura et al., Icarus 157, 349-361, 2002).

\author{
Philippe Lamy \\ President of the Commission
}

